\title{
Bismuth vanadium oxide can promote the growth and activity of
} roots in Arabidopsis thaliana

Cong Gao, Shuai Lu, Yongzhou Wang, Hao Xu, Xiaoxiao Gao, Yiwen Gu, Hongyun Xuan, Baohua Wang*, Huihua Yuan* and Yunying Cao*

College of Life Sciences, Nantong University, Nantong, Jiangsu, China; GC1909310005@sohu.com（C.G); 2008310011@stmail.ntu.edu.cn（S.L); wangzizhouzi@163.com(Y.W); haoxu66666@163.com (H.X.) gyw20000102@126.com(Y.G); g13160495899@163.com（X.＜wide>G) hyxuan_seu@163.com (H.X); bhwang@ntu.edu.cn (B.W); yuanhh@ntu.edu.cn (Y.H); cyy@ntu.edu.cn (Y.C);

*Correspondence: cyy@ntu.edu.cn; yuanhh@ntu.edu.cn; bhwang@ntu.edu.cn Tel.: +86-0513-85012827

Abstract: The excellent properties of nanomaterials have been confirmed in many fields, but the effects on plants are still unclear. In this study, we have found that bismuth vanadium promoted the growth of taproots and the development of lateral roots, increased the roots activity by regulating the expression of polyamine or hormone-related genes and reducing the accumulation of reactive oxygen species in Arabisdopsis thaliana. Meantime, BV had higher antimicrobial effect than that of graphene oxide (GO). These results showed us a new perspective possibility of nanomaterials assisting the development of plants and crops, which is expected to become one of the ways to solve the problem of controlling and promoting the development of plants and crops.

Keywords: bismuth vanadium oxide; roots activity; reactive oxygen generation ; gene expression 


\section{INTRODUCTION}

Compared with traditional materials, nanomaterials have many advantages, and are applied in the fields of information industry, environmental industry, energy and environmental protection, biological medicine and other fields [1]. The growth and development of plants are regulated by many factors, such as temperature and hormone [2-6]. Meanwhile, more and more studies show that nanomaterials also have an impact on the growth and development of plants. For example, modified polystyrene nanomaterials with different polarity could inhibit the roots development of Arabidopsis [7]. Copper oxide nanoparticles regulated the phenotype of mung bean [8]. CuONPs can decrease the mitotic index of onion roots tip gradually and increase the abnormal index [9]. Furthermore, the effects of nano- $\mathrm{TiO}_{2}$ foliage intervention on cadmium bioaccumulation, stress kinase and potential dietary health risks in cowpea plants were also reported [10]. What's more, the lignification degree of xylem in roots and stems of fenugreek treated with nanosilicon materials was significantly higher than that of control plants [11]. Most of the nanomaterials above showed toxic effects on plants, however, some of them showed positive role. A research reported that graphene oxide $(\mathrm{GO})$ promoted the growth of watermelon, including increasing roots length, leaf area, leaf number and flower bud formation [12]. Multi-walled carbon nanotubes (MWCNTs) could stimulate the seed germination of three important crops (barley, soybean and maize), enhance the root length in the seedlings of Phaseolus mungo and the germination index of Brassica juncea in low concentration [13-15]. However, its application in plant related fields is still less, and due to the variety and different characteristics of nanomaterials, it also shows different effects on plants.

Metal vanadate have been widely used for potential applications as batteries, implantable cardiac defibrillators, and photo catalysis [16]. Specially, bismuth 
vanadate nanomaterials (herein after referred to as BV) has emerged as a promising candidate due to its unique nontoxicity, chemical stability, optical, and ferro-elastic properties [17]. Various applications of BV has been well studied in eco-friendly yellow pigment, water splitting, sensors, pollutant degradation. Recently, BV has been drawn great attation in biological applications. The BV composite material exhibited excellent potential for inactivation of Escherichia coli [18-20]. These works provided evidence about the positive effects of the BV nanomaterials on microbiome. However, currently no or little attempts have been made to explore the effect of BV nanomaterials on plants. Therefore, the objective of this study was to use Arabidopsis thaliana as a model plant to determine the effect of BV on some aspects including the length of taproot, the number of lateral roots, the activity of plant root and superoxide anion $\left(\mathrm{O}_{2}{ }^{-}\right)$generation, the antimicrobial activity and the expression of related genes (Figure 1). This evaluation was based on the effects of applying different concentrations of the $\mathrm{BV}$ on root elongation rate, so as to clarify the effect of $\mathrm{BV}$ on root in Arabidopsis thaliana.

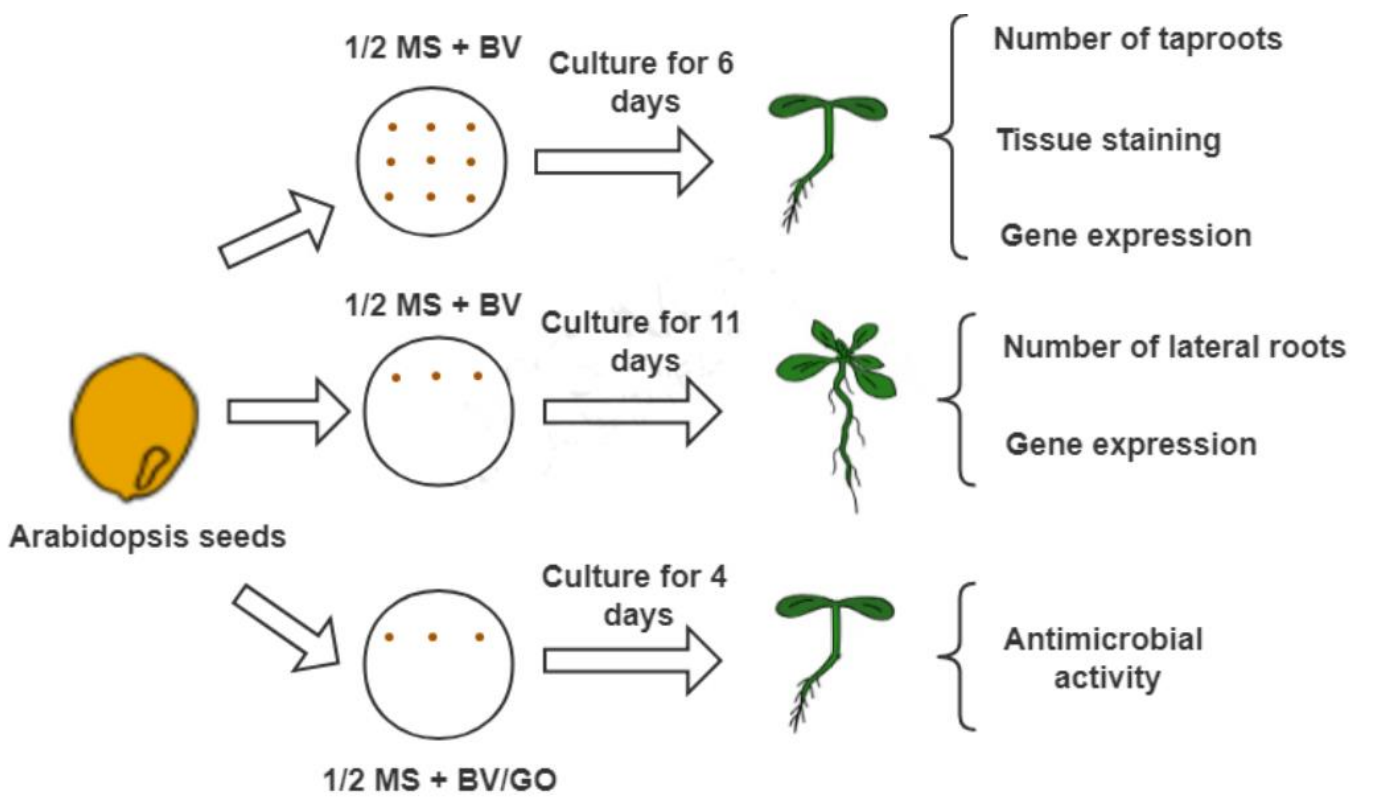

Figure 1. Workflow for BV on the root growth experiment in Arabidopsis thaliana. 


\section{MATERIALS AND METHODS}

\section{Plant material}

Seeds of Arabidopsis thaliana L., Columbia-0 (Col-0) were surface-sterilized with $70 \%$ ethanol and $20 \%$ bleach. Materials were grown at $22{ }^{\circ} \mathrm{C} / 20{ }^{\circ} \mathrm{C}$ under a photoperiod with $16 \mathrm{~h}$ of light and $8 \mathrm{~h}$ of dark and a light intensity of $100 \mu \mathrm{mol} \cdot \mathrm{m}$ ${ }^{-2} \cdot \mathrm{s}^{-1}$ conditions in an incubator (QY-14; Nanjing Quanyou Electronic Technology Co., Ltd, China).

\section{Preparation and characterization of BV}

$\mathrm{Bi}\left(\mathrm{NO}_{3}\right)_{3} \cdot 5 \mathrm{H}_{2} \mathrm{O}(2.1830 \mathrm{~g})$ and EDTA $(4 \mathrm{~g})$ were dissolved in dilute $\mathrm{HNO}_{3}$ solution $(50 \mathrm{~mL}, 2 \mathrm{mM})$, and stirred at $90{ }^{\circ} \mathrm{C}$ for $30 \mathrm{~min}(200 \mathrm{rpm})$ to get Solution A. $\mathrm{NH}_{4} \mathrm{VO}_{3}(0.5260 \mathrm{~g})$ was dissolved in deionized water $(50 \mathrm{~mL})$ at $60{ }^{\circ} \mathrm{C}$ to obtain Solution B. Then, solution B was mixed with solution A, and the $\mathrm{pH}$ was adjusted to 7 by $\mathrm{NH}_{4} \mathrm{OH}$. The above mixed solution was stirred for $1 \mathrm{~h}$ at $50{ }^{\circ} \mathrm{C}$, poured into a 150 $\mathrm{mL}$ Teflon-lined stainless-steel autoclave, and maintained at $180{ }^{\circ} \mathrm{C}$ for $6 \mathrm{~h}$. The prepared precipitate was washed with ethanol and deionized water for several times, and dried in a vacuum oven at $75{ }^{\circ} \mathrm{C}$ for overnight to formate $\mathrm{BV}$ nanomaterials. Sample morphology and surface elemental composition was examined by scanning electron microscopy (SEM; JSM6510, JEOL, Japan) coupled with energy-dispersive spectrometry $(\mathrm{EDS})$ at an accelerating voltage of $10 \mathrm{kV}$. X-ray diffraction (XRD; Ultima IV, Rigaku, Japan) patterns of samples were recorded by using high-intensity $\mathrm{Cu}$ Ka radiation $(\lambda=0.154 \mathrm{~nm})$ in the range of $2 \theta=10-80^{\circ}$ at a scan rate of $5^{\circ} \cdot \mathrm{min}^{-1}$.

\section{Preparation of roots for analysis}

With respect to plants grown in solid media, A. thaliana seeds were germinated 
on a square plate $(10 \mathrm{~cm} \times 10 \mathrm{~cm})$ that contained sterilized solidified half-strength MS (Sigma-Aldrich, St. Louis, MO, USA) media that consisted of $0.8 \%$ agar (Affymetrix, Inc. Cleveland, Ohio, USA) and 1\% sucrose. $10 \mathrm{~g} \mathrm{BV}$ powder was dissolved in $1 \mathrm{~L}$ of deionized water and sterilized in an autoclave (SANYO Labo Autoclave, MLS-3020). BV solution that had been sonicated for $1 \mathrm{~h}$ was blended with the sterilized half-strength MS medium by stirring with $0,20,50,100$ and 200 $\mu \mathrm{g} \cdot \mathrm{mL}^{-1}$ nanomaterials, and the resulting medium (here in after referred to as the BV/MS medium). Totally 30-40 seeds were planted in the BV/MS medium and placed in $4{ }^{\circ} \mathrm{C}$ for two days and then transferred to a growth chamber as described above. The roots were imaged with a scanner, and the primary root length was measured using ImageJ software (National Institutes of Health, United States) after six days of exposure. The lateral root number was analysed after eleven days of exposure. The roots of the plants exposed to nanomaterials for 6 days were stained with propidium iodide for viability testing meristem, extension zone length, tip diameter and rootcap size of primary root as described previously [21].

\section{Detection of superoxide anion radical $\mathrm{O}_{2} \cdot{ }^{-}$and root activity}

To visualize $\mathrm{O}_{2} .^{-}$and root activity in plant roots in situ, nitroblue tetrazolium (NBT) and 2,3,5-Triphenyl tetrazolium chloride (TTC) staining were performed, respectively, as described by previously $[22,23]$ and modified slightly. The root $\mathrm{O}_{2} \cdot^{-}$ content was measured by incubating the plants in $1 \%$ NBT within $20 \mathrm{mM}$ K-phosphate, then washed with distilled water, decolorized with $70 \%$ ethanol solution in $90{ }^{\circ} \mathrm{C}$ water for $20 \mathrm{~min}$. The root activity was measured by incubating the plants in 
$2 \%$ TTC at $37{ }^{\circ} \mathrm{C}$ for $5 \mathrm{~h}$. Images of the plants or roots were obtained under brightfield illumination.

\section{Antimicrobial effect of nanomaterials}

In order to clear the antimicrobial effect of nanomaterials, the seeds of A. thaliana were cultured in half-strength MS media with adding 0, 50, 100, 200 $\mu \mathrm{g} \cdot \mathrm{mL}^{-1}$ non-autoclaved nanomaterials and analysed the microbial number after $4 \mathrm{~d}$.

\section{RT-qPCR analysis}

Plants were grown on half-strength MS media with either BV $\left(200 \mu \mathrm{g} \cdot \mathrm{mL}^{-1}\right)$ or without nanomaterials. The primary root about six-day-old and all the roots about eleven-day-old were harvested. Total RNA was isolated using TRIzol reagent (Invitrogen) and converted to complementary DNA (cDNA) using a Transcriptor First Strand cDNA Synthesis Kit (Roche) following the manufacturer's protocol. qPCR was performed using a 7500 Real-time PCR Detection System (Bio-Rad) in conjunction with Fast Start Universal SYBR Green Master Mix (Roche). ACT2 (At3G18780) was used as a reference gene. The primers used for qPCR are listed in Table S1.

\section{Statistical analyses}

Without special instructions, all experiments were repeated at least three times. SPSS 20.0 and SigmaPlot 10.0 were used for statistical analysis and plotting. Statistical differences were analysed by Duncan test. The data were considered significant in accordance with the following criteria $(\mathrm{P}<0.05)$. 


\section{RESULT AND DICUSSION}

\section{Fabrication and Characterization of nanomaterial}

The morphology and structures of the as-prepared BV nanomaterials were detected by SEM (Figure 2). Geometrically shaped-like nanoparticles are observed for BV (Figure 2A). In addition, elements of C, O, Bi, and V were clearly displayed in the the EDS spectra (Figure 2B), conforming the presence of BV nanomaterials. The phase purities and crystallinities of the BV nanomaterials was further characterized by XRD analysis (Figure 2C). Obviously, the diffraction peaks at $2 \theta=18.9^{\circ}, 19.3^{\circ}, 29.3^{\circ}$, $30.9^{\circ}$, and $53.6^{\circ}$ revealed the (110), (011), (121), (040), and (161) planes for BV nanomaterials in the XRD pattern, indexed to monoclinic scheelite-type BV (JCPDS no. 14-0688).

A

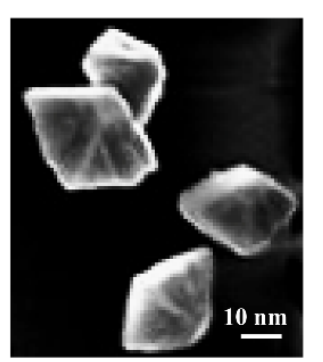

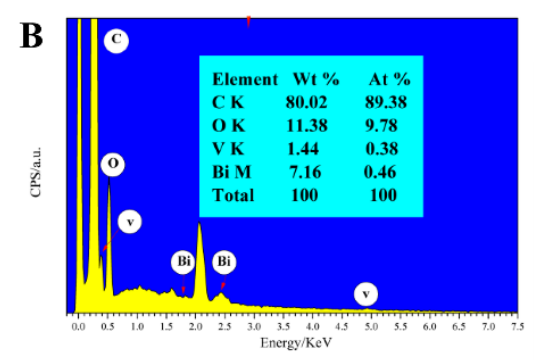

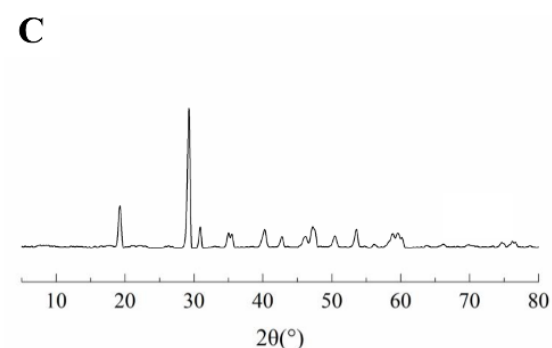

Figure.2 Characterization of BV. SEM (A), EDS spectra (B), and XRD patterns (C) images of of BV

\section{Effects of nanomaterial on Arabidopsis roots}

To confirm the influence of $\mathrm{BV}$ on the primary root length and lateral root numbers, these two traits were evaluated after 6 and 11 days of plant growth on the various BV/MS media. Figure 3 shows that nanomaterial has effects on the length of primary roots in Arabidopsis, and the different concentration of nanomaterials had 
inconsistent changes. Compared with the control, 20, 50 and $100 \mu \mathrm{g} \cdot \mathrm{mL}^{-1} \mathrm{BV}$ significantly reduced the length of taproot about $13.0 \sim 20.0 \%$, while $200 \mu \mathrm{g} \cdot \mathrm{mL}^{-1} \mathrm{BV}$ remarkably enhanced the length about $49.0 \%$. This may imply that BV has a dual effect on the length of plant roots. This phenomenon of dual effects on plants is not the first time, and a report showed that GO had a similar phenomenon [12]. There may also be another situation, that is, it is related to the concentration of BV. This hypothesis is based on the fact that BV is not detected on the surface of the treated roots observed by EDS (Figure S1). This implies that BV plays a role after entering the cells. Different from animal cells, plant cells have cell walls and almost no phagocytosis. Before entering plant cells, nanomaterials must penetrate cell walls and plasma membranes. When the concentration of BV is low, it can not be well absorbed by plant roots, so it can not play a role. When the concentration reaches a certain value, BV can be absorbed by the roots and works.
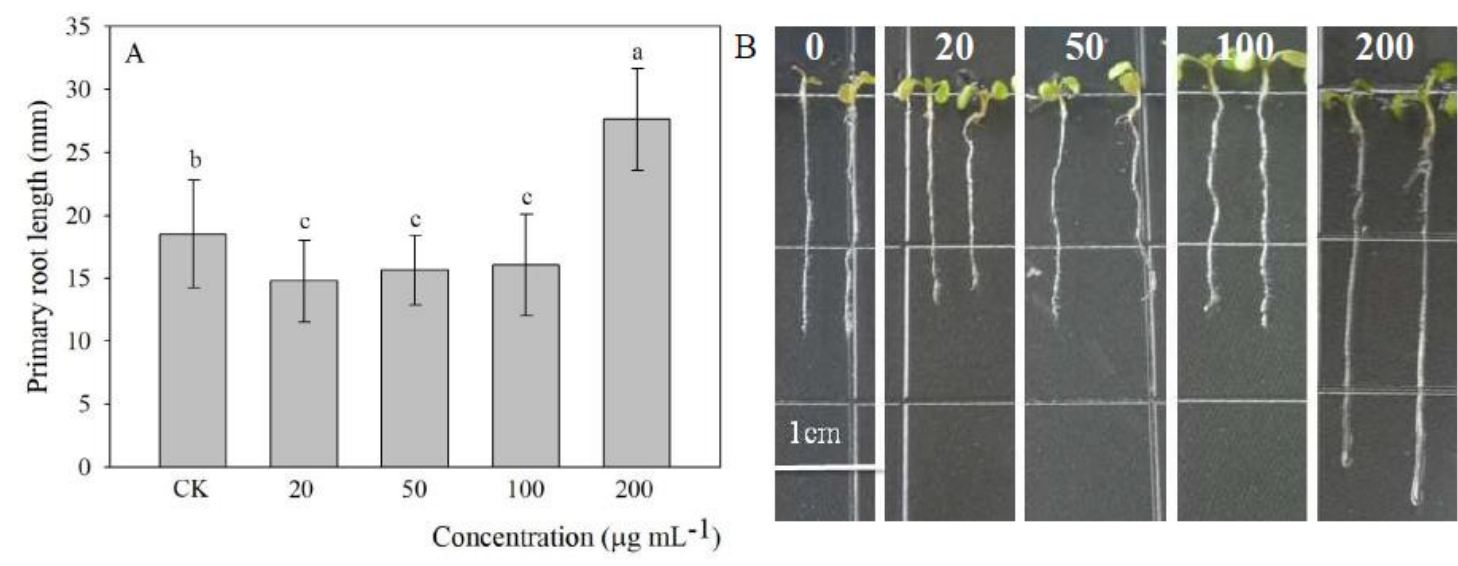

Figure 3. Effects of different concentrations of nanomaterial BV on taproot length of Arabidopsis.

Scale bars, $1 \mathrm{~cm} .0,20,50,100,200$ of figure B were added to MS medium with 0 , 20. $50,100,200 \mu \mathrm{g} \cdot \mathrm{mL}^{-1}$ of $\mathrm{BV}$, respectively. $\mathrm{N}=30$. 
In addition, we also observed that the addition of nanomaterial BV can also affect the lateral roots number (Figure 4). BV treatment of different concentration increased the number of lateral roots of all the plants, and the difference of 20 $\mu \mathrm{g} \cdot \mathrm{mL}^{-1}$ and $50 \mu \mathrm{g} \cdot \mathrm{mL}^{-1}$ treatments was at a significantly level. There was no significant difference among the other treatments compared to the treatment without adding nanomaterial. In general, the effect of low concentration was more beneficial to increase the number of lateral roots. Previous studies have shown that the development of lateral roots can be affected by many kinds of plant hormones [24]. In our study, the effect of nanomaterial BV on lateral roots may be achieved by changing plant hormones. The lateral root is connected with the taproot, and the water and nutrient can flow through the catheter and sieve tube. Generally speaking, the growth of lateral roots was inhibited by taproot, especially near the root tip. To a certain extent, BV treatment was in line with this trend. It is necessary to further study these phenomena, and the cause of these different influence modes can increase our understanding of how BV affects plants.
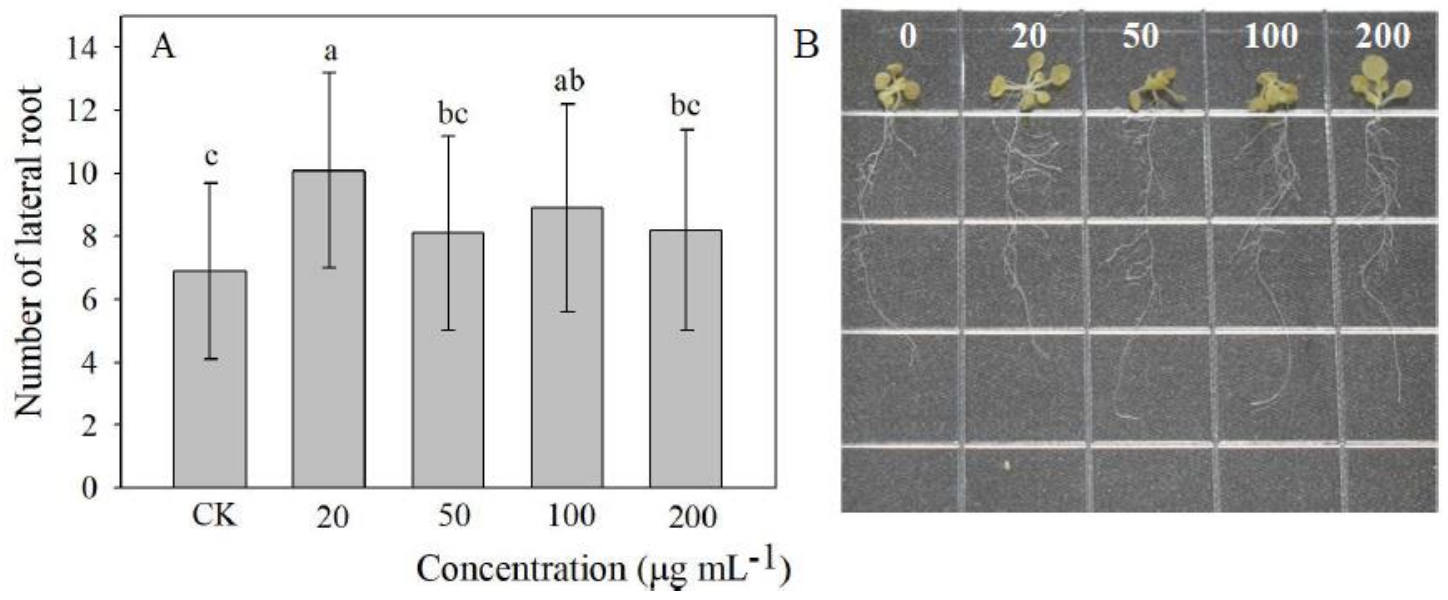

Figure 4. Effects of different concentrations of nanomaterial BV on the number of lateral roots in Arabidopsis. 
0, 20, 50,100, 200 of figure B were added to MS medium with 0, 20. 50,100, 200 $\mu \mathrm{g} \cdot \mathrm{mL}^{-1}$ of $\mathrm{BV}$, respectively. $\mathrm{N}=15$.

Based on the fact that it had been found that BV promoted the development of taproot, the influence of nanomaterial BV by measuring the different position of root was futher explored (Figure 5). BV treatment enhanced the length of extension zone, but there is little effect on that of meristem zone and taproot cap. Therefore, the result suggested that BV might have an effect on the extension zone and lead to the increase of the length of the taproot. Futhermore, the diameter of the primary root was also analysed. Unfortunately, there was no significant change in diameter, suggesting that BV has limited effect on root diameter.
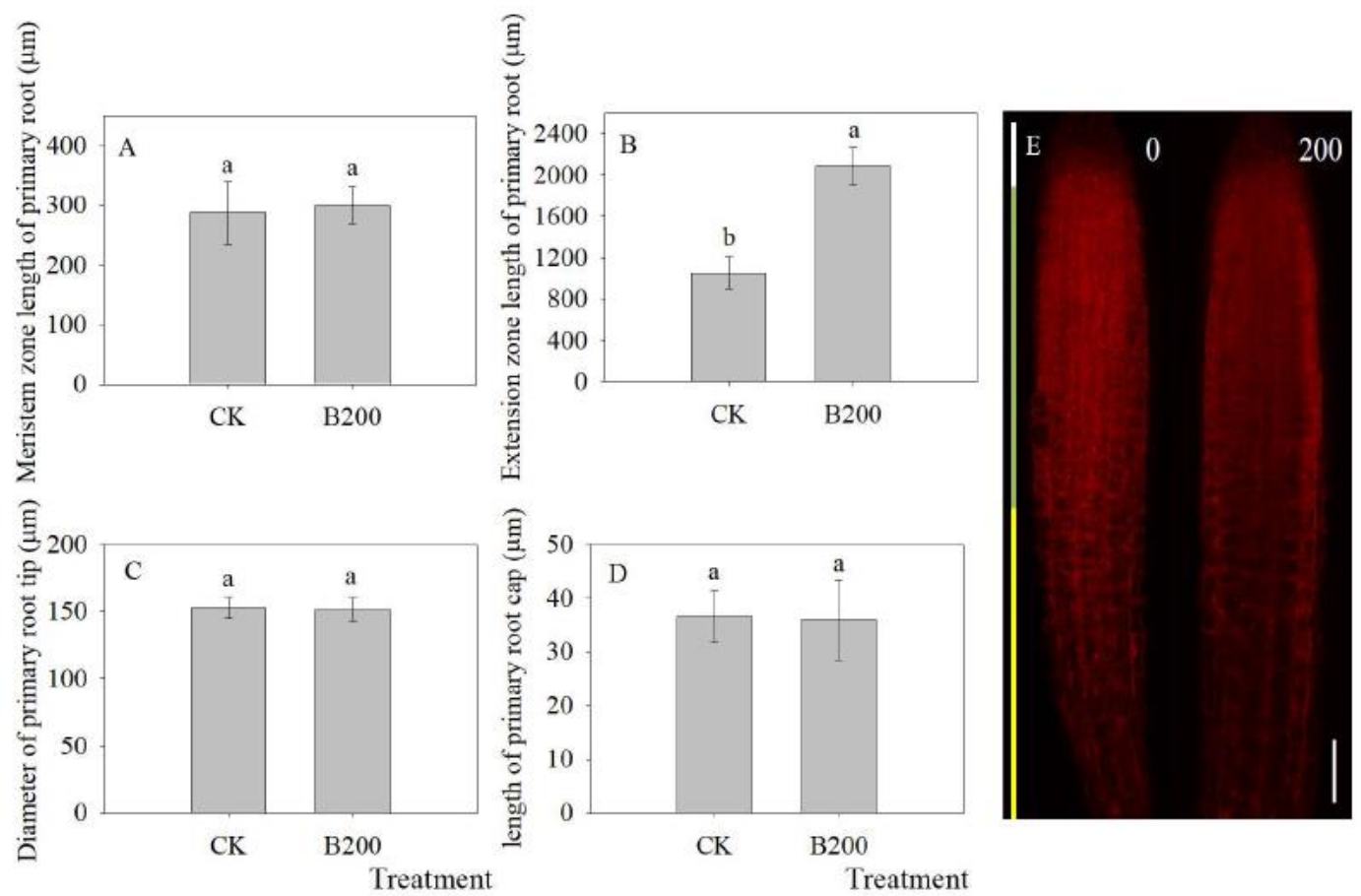

Figure 5. Effect of nanomaterial BV on primary root length in Arabidopsis.

A: meristem zone length of primary root. B: extension zone length of primary root root. C: the diameter of root tip. D. the length of taproot cap. E: Representative images of roots stained with propidium iodide to outline the living cells, showing the 
root cap, the meristem domain and the maturation zone after four days of incubation, Scale bars, $50 \mu \mathrm{m}$. CK: the control without adding $\mathrm{BiVO}_{4}$; B200: $200 \mu \mathrm{g} \cdot \mathrm{mL}^{-1} \mathrm{BV}$ treatment. $\mathrm{N}=30$.

\section{The root activity and $\mathrm{O}_{2} \cdot-$ production analysis}

The above experiments had shown that BV promoted roots development in plants. Therefore, we tried to make clear whether root activity and $\mathrm{O}_{2} .^{-}$also changed. $\mathrm{O}_{2} \cdot-$ belongs to the reactive oxygen species group, which has strong oxidizability. It plays an important role in the process of physical reaction [25]. When organisms are stressed, the production of reactive oxygen species in the body will increase greatly; when it exceeds the antioxidant defense capacity of organisms, cellular components such as lipids, proteins, nucleic acids and so on will be irreversibly damaged under the action of $\mathrm{O}_{2} \cdot{ }^{-}$to interfere with cell metabolism or cause cell death [26]. Succinic acid is a key intermediate in the tricarboxylic acid cycle. Under physiological conditions, succinate dehydrogenase in mitochondria oxidizes succinate to fumaric acid and releases electrons to participate in oxidative phosphorylation [27]. The reduction of TTC indicates the activity of dehydrogenase can be used as an indicator of root activity. Consequently, TTC and NBT staining were performed (Figure 6).

As a method to identify roots vitality, TTC staining is based on the principle that living tissue can produce hydrogen ions under the action of dehydrogenase and has reduction ability. The depth of tissue coloration indicate the strength of root activity. After growing in half-strength MS medium for 6 days, we found that the roots tip color of $200 \mu \mathrm{g} \cdot \mathrm{mL}^{-1} \mathrm{BV}$ was significantly darker than that of the control, indicating 
that the seedlings treated with appropriate concentration of nanomaterials increased the roots activity of Arabidopsis. Xie et al also reported that plant root activity and the plant root growth were positive related under GO treatment in napus seedlings [28]. This suggests that BV can affect the tricarboxylic acid cycle and plays a positive role in enhancing plant activity.

Superoxide dismutase (SOD) is an enzyme that scavenges $\mathrm{O}_{2}^{-} \cdot \mathrm{O}_{2}^{-}$can reduce NBT to blue methylhydrazone, so SOD inhibits the formation of methylhydrazone. The deeper the blue color of the tissue treated with NBT solution, the lower the enzyme activity. Conversely, the higher the enzyme activity. Therefore, this method can be used to measure $\mathrm{O}_{2}^{-}$. In this experiment, we found that Arabidopsis seedlings of $200 \mu \mathrm{g} \cdot \mathrm{mL}^{-1} \mathrm{BV}$ was slightly lighter than that of the control, indicating that the accumulation of $\mathrm{O}_{2}^{-}$in seedlings was reduced by appropriate concentration of $\mathrm{BV}$. The plants respond to nanoparticles mediated stresses through reactive oxygen species generation [29]. It is widely accepted that the toxicity of nanoparticles on plants is commonly evident at high concentrations and attributed to the generation of reactive oxygen species (ROS) [30]. But a report showded that SOD activity was widely stimulated after the exposure of plants to $\mathrm{TiO}_{2}$ NPs and reduced the level of total ROS. Our data are consistent with the results [31]. It suggested that BV enhance the SOD activity and promoted the plant growth by inducing the accumulation of ROS. 
A
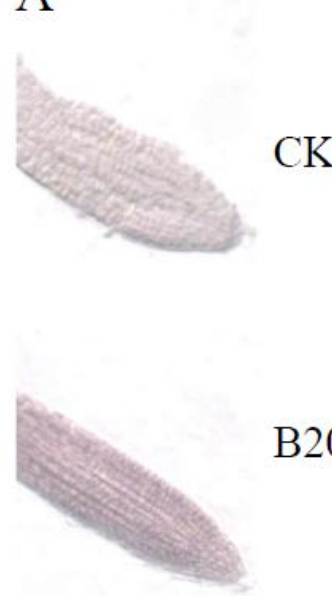

B200

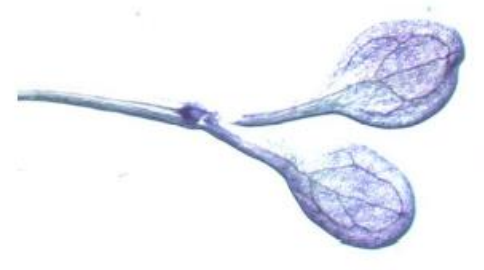

Figure 6. Effect of nanomaterial BV treatment on Arabidopsis tissue staining. A:TTC staining, Scale bar, 5mm; B: NBT staining, Scale bar, $2 \mathrm{~mm}$. CK: the control without nanomaterial BV; B200: $200 \mu \mathrm{g} \cdot \mathrm{mL}^{-1} \mathrm{BiVO}_{4}$ treatment. $\mathrm{N}=6$.

\section{Antimicrobial effect of nanomaterials}

In view of the reported antimicrobial activity of BV [32], the antimicrobial experiments of BV were done (Figure 7). Graphene oxide (GO) with certain antimicrobial activity [33-35] was selected as the control to compare the antimicrobial effect of BV. Adding different concentrations of nanomaterial unsterilized BV into half-strength MS medium, it was found that the infection degree of BV was lower than that of GO after 4 days of culture. There was no significant difference between 100 and $200 \mu \mathrm{g} \cdot \mathrm{mL}^{-1} \mathrm{BV}$ treatment, but both were significantly higher than 50 $\mu \mathrm{g} \cdot \mathrm{mL}^{-1}$ treatment. This indicates that $\mathrm{BV}$ has certain antimicrobial activity. However, with the increase of GO concentration, the species, number and size of infected microbe increased significantly (Figure 7). It can also be seen from the figure that the 
growth of Arabidopsis in the medium added BV is better than that in the medium added GO. These results indicate that the two nanomaterials have different resistance to microbe, the antimicrobial activity of BV was better than that of GO. At the same time, the antimcrobial property of BV also gives the possibility to develop in more fields, such as plant tissue culture and other experiments in which adding an appropriate amount of BV can play a certain antimicrobial effect. Interestingly, this result can also be used to explain why low concentrations inhibit root development and high concentrations promote root development. Based on the bacteriostatic effect of BV, when it can not be absorbed by roots, it may inhibit the development of roots when it is free outside the roots.
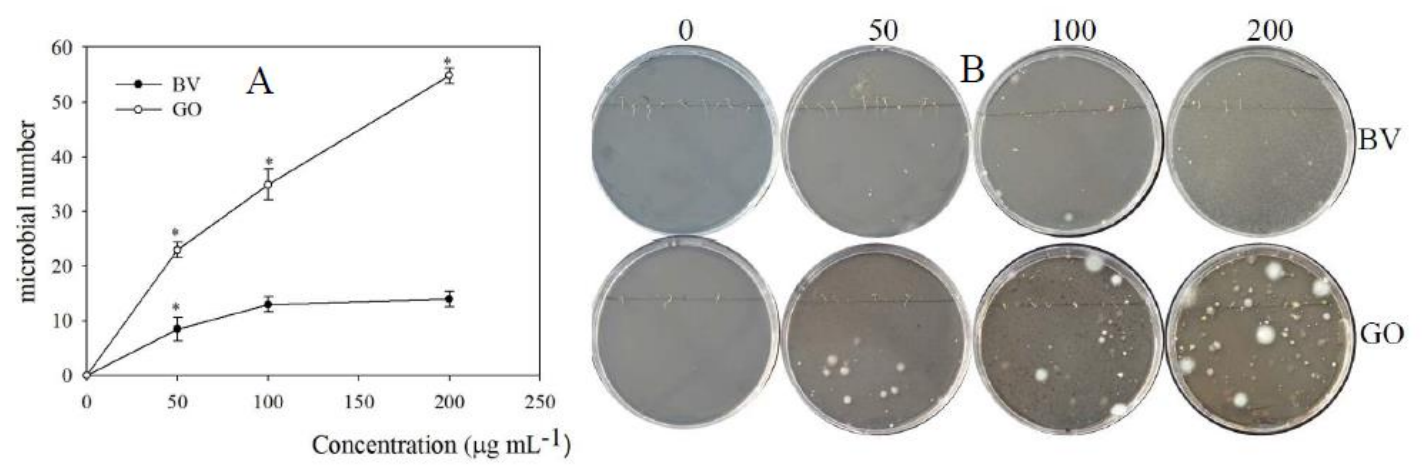

Figure 7. Antimicrobial effect of nanomaterials BV and GO on Arabidopsis.

B: Representative images of without sterilized nanomaterials of different concentration. $0,50,100,2000,20,50,100,200$ of figure B were added to MS medium with $0,20,50,100,200 \mu \mathrm{g} \cdot \mathrm{mL}^{-1}$ of $\mathrm{BV}$ or $\mathrm{GO}$, respectively. $\mathrm{BV}$ : $\mathrm{BV}$ treatment; GO: treatment. $\mathrm{N}=3$

\section{Changes of gene expression related to root development in Arabidopsis}

Now that we have found nanomaterial BV can change root length and lateral root number, some genes related to root development are also further investigated. 
Therefore, we selected three types of genes, among which two genes mainly affected the primary roots $(A D C 1, D A R 2)$, two genes mainly affected the lateral root ( $A R F 19$, $C K X 1)$, and two genes affected both (ERF II, IQM3) (Figure 8). Surprisingly, in the primary roots, $A D C 1, D A R 2, E R F I I$ and IQM3 show the same trend. ADC1 can affect the synthesis of polyamines in Arabidopsis [36], and studies have shown that polyamines can regulate root length [37]. DAR2 can be associated with a variety of hormones, and then affect the development of root meristem [38]. ERF II, as a transcription factor, is also regulated by hormones, thus affecting the development of root [39]. In Arabidopsis, IQM family belongs to calmodulin binding protein family with IQ motif. IQM3 is involved in the regulation of plant root development [40]. After treatment with $\mathrm{BV}, E R F I I$ and IQM3 were significantly up-regulated. In general, the expression levels of the four genes were significantly up-regulated. These results suggest that $\mathrm{BV}$ may be involved in the regulation of plant taproot development in a variety of ways.

Furthermore, in the lateral root, the related genes show a more complex phenomenon. $A R F 19$, as a gene mediating hormone regulation of lateral roots, is the most in-depth and clear regulatory pathway [41]. In the experiment, BV treatment increased significantly the expression of $A R F 19$. $C K X 1$ could mediate cytokinin to regulate lateral root development, which was significantly down-regulated after BV treatment. Moreover, IQM3 shows a similar level between BV treatment and the control. ERF II was significantly up-regulated in root treated with BV. All kinds of signs showed that BV can regulat root development by mediating hormones. 

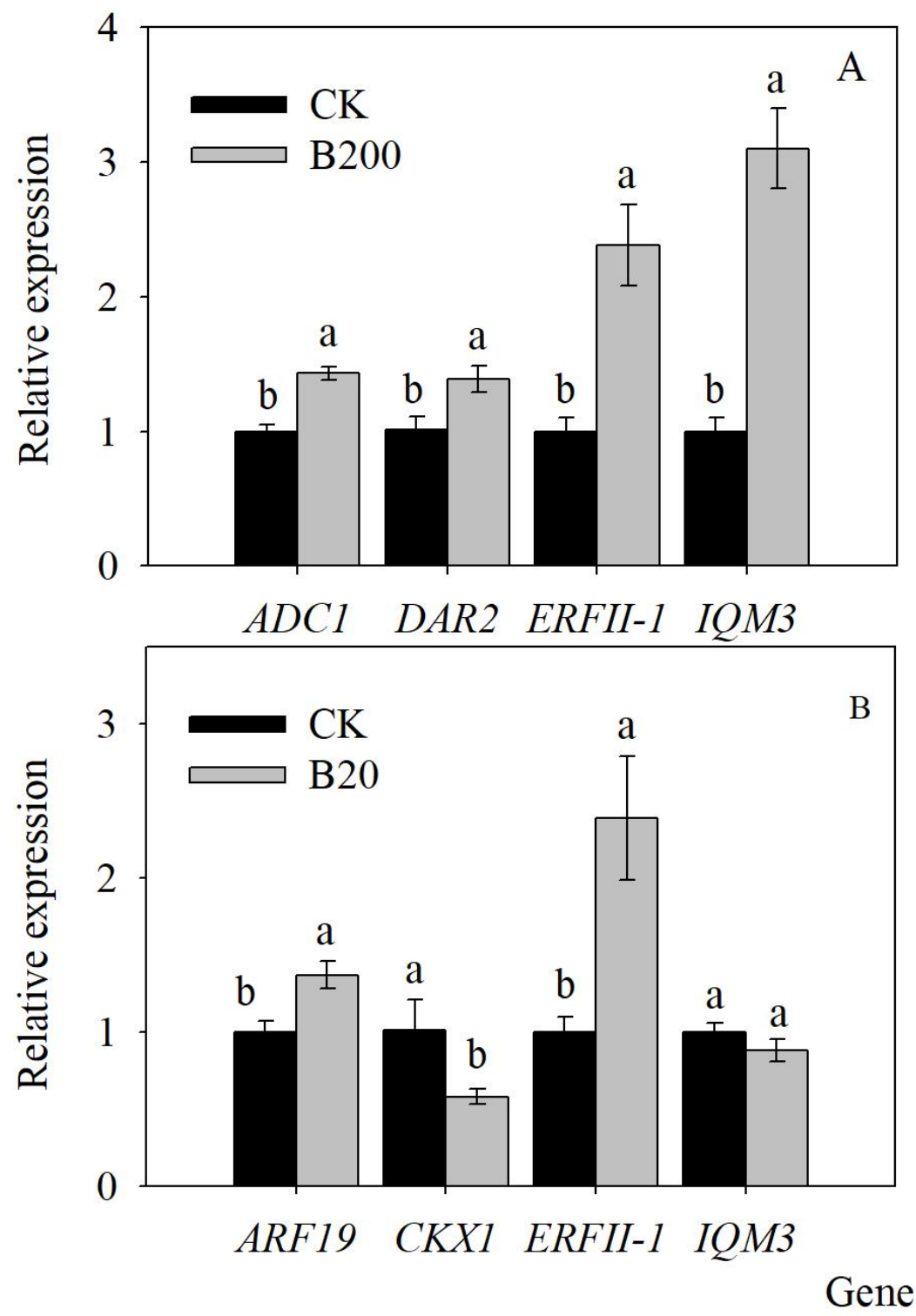

Figure 8. Effect of nanomaterial $\mathrm{BV}$ on relative gene expression of primary root length and lateral root numbers in Arabidopsis.

A: relative gene expression of primary root length. B: relative gene expression of lateral root number. CK: without nanomaterial treatment. B20 and B200 was treated with BV about $20 \mu \mathrm{g} \cdot \mathrm{ml}^{-1}$ and $200 \mu \mathrm{g} \cdot \mathrm{ml}^{-1} . \mathrm{N}=3$

\section{CONCLUSION}

In our study, BV can promote the roots development of Arabidopsis by 
increasing the length of taproots and the number of lateral roots. The results of tissue staining showed that BV played a positive role and enhanced plant root vitality. What's more, BV showed better antimicrobial activity than GO. In addition, it proved that the expression level of root development related genes also changed. In conclusion, proper concentrations of $\mathrm{BV}$ are expected to be used as promoters for plant growth and development. BV is expected to be widely used in plant related fields due to its excellent properties, low production cost and antimicrobial properties. However, there are many problems to be solved. For example, only Arabidopsis is used as experimetal materials in this research, whereas how BV affects other plants remains to be explored. Finally, it is necessary to explore the mechanism of nanomaterials affecting plants, and deepen the understanding of them.

\section{Supplementary Materials}

Table S1 : Primers used for qRT-PCR

Figure S1: Effect of nanomaterial BV on the extension zone of taproot in Arabidopsis.

Acknowledgments: This work was carried out with the support of the "Jiangsu Province Government Project (BM2018003), the open funds of the Jiangsu Key Laboratory of Crop Genomics and Molecular Breeding (PL201805), the National Natural Science Foundation of China (81801856 and 32000965), the Natural Science Foundation of Jiangsu Province (BK20180949 and BK20200964), the Six Talent Peaks Project in Jiangsu Province (XCL-063), the Project of Natural Science Research of Higher Education Institutions of Jiangsu Province (20KJB430042), 
National Undergraduate Innovation and Entrepreneurship Training

Program of China (202010304053Z).”

Author Contributions: Conceptualization, Baohua Wang, Huihua Yuan and Yunying

Cao; Data curation, Cong Gao, Shuai Lu, Yongzhou Wang, Hao Xu, Xiaoxiao Gao, Yiwen Gu and Hongyun Xuan; Funding acquisition, Huihua Yuan and Yunying Cao; Writing - original draft, Cong Gao; Writing - review \& editing, Baohua Wang, Huihua Yuan and Yunying Cao; Baohua Wang, Huihua Yuan and Yunying Cao take full responsibility for this manuscript. All authors have read and agreed to the published version of the manuscript.

Conflicts of Interest: The authors declare no conflict of interest.

\section{References}

1. Castiglione, M.R.; Giorgetti, L.; Geri, C.; Cremonini, R. The effects of nano- $\mathrm{TiO}_{2}$ on seed germination, development and mitosis of root tip cells of Vicia narbonensis L. and Zea mays L. J. Nanopart. Res. 2011, 13, 2443-2449.

2. Kim, J. Y.; Song, J. T.; Seo, H. S. COP1 regulates plant growth and development in response to light at the post-translational level. J. Exp. Bot. 2017, 68, 4737-4748.

3. Brandhoff, B.; Simon, A.; Dornieden, A, Schumacher, J. Regulation of conidiation in Botrytis cinerea involves the light-responsive transcriptional regulators BcLTF3 and BcREG1. Curr. Genet. 2017, 63, 931-949.

4. Gómez-Merino FC, Trejo-Téllez LI, García-Jiménez A, Escobar-Sepúlveda HF, Ramírez-Olvera SM. Silicon flow from root to shoot in pepper: a comprehensive in silico analysis reveals a potential linkage between gene expression and hormone signaling that stimulates plant growth and metabolism. Peer J. 2020, 8, ee10053.

5. Sun, W. J.; Ma, Z. T.; Chen, H.; Liu M. MYB gene family in potato (Solanum tuberosum L.): Genome-wide identification of hormone-responsive reveals their potential functions in growth and development. Int. J. Mol. Sci. 2019, 20, 4847.

6. Ibañez, C.; Poeschl, Y.; Peterson, T.; Bellstädt, J.; Denk, K.; Gogol-Döring, A.; Quint, M.; Delker, C. Ambient temperature and genotype differentially affect developmental and phenotypic plasticity in Arabidopsis thaliana. BMC plant biology. 2017, 17, 114. 
7. Sun, X.; Yuan, X.; Jia, Y.; Feng, L.; Zhu, F.; Dong, S.; Liu, J.; Kong, X.; Tian, H.; Duan, J.; Ding, Z.; Wang, S.; Xing, B. Differentially charged nanoplastics demonstrate distinct accumulation in Arabidopsis thaliana. Nat. Nanotechnol. 2020, 15, 755-760.

8. Nair, P.M.G.; Kim, S.; Chung, I.M. Copper oxide nanoparticle toxicity in mung bean ( Vigna radiata L.) seedlings: physiological and molecular level responses of in vitro grown plants. Acta Physiol. Plant. 2014, 36, 2947-2958.

9. Nagaonkar, D.; Shende, S.; Rai, M. Biosynthesis of copper nanoparticles and its effect on actively dividing cells of mitosis in Allium cepa. Biotechnol. Prog. 2015, 31, 557-565.

10. Ogunkunle, C.O.; Odulaja, D.A.; Akande, F.O.; Varun, M.; Vishwakarma, V.; Fatoba, P.O. Cadmium toxicity in cowpea plant: Effect of foliar intervention of nano- $\mathrm{TiO}_{2}$ on tissue $\mathrm{Cd}$ bioaccumulation, stress enzymes and potential dietary health risk. J. Biotechnol. 2020, 310, 54-61.

11. Nazaralian, S.; Majd, A.; Irian, S.; Najafi, F.; Ghahremaninejad, F.; Landberg, T.; Greger, M. Comparison of silicon nanoparticles and silicate treatments in fenugreek. Plant Physiol. Biochem. 2017, 115, 25-33.

12. Park, S.; Choi, K.S.; Kim, S.; Gwon, Y.; Kim, J. Graphene oxide-assisted promotion of plant growth and stability. Nanomaterials. 2020, 10, 758 .

13. Lahiani, M.H.; Dervishi, E.; Chen, J.; Nima, Z.; Gaume, A.; Biris, A.S.; Khodakovskaya, M.V. Impact of carbon nanotube exposure to seeds of valuable crops. ACS Appl. Mater. Interfaces. 2013, 5, 7965-7973.

14. Ghodake, G.; Seo, Y.D.; Park, D.; Lee, D.S. Phytotoxicity of carbon nanotubes assessed by Brassica Juncea and Phaseolus Mungo. J. Nanoelectron. Optoelectron. 2010, 5, 157-160.

15. Mondal, A.; Basu, R.; Das, S.; Nandy, P. Beneficial role of carbon nanotubes on mustard plant growth: an agricultural prospect. J. Nanopart. Res. 2011, 13, 4519-4528.

16. Sivakumar, V.; Suresh, R.; Giribabu, K.; Narayanan, V. $\mathrm{BiVO}_{4}$ nanoparticles: Preparation, characterization and photocatalytic activity. Cogent Chem. 2015, 1, 1074647.

17. Sarkar, S.; Chattopadhyay, K.K. Size-dependent optical and dielectric properties of $\mathrm{BiVO}_{4}$ nanocrystals. Physica E. 2012, 44, 1742-1746.

18. Guan, D.; Niu, C.; Wen, X.; Guo, H.; Deng, C.; Zeng, G. Enhanced Escherichia coli inactivation and oxytetracycline hydrochloride degradation by a Z-scheme silver iodide decorated bismuth vanadate nanocomposite under visible light irradiation. J Colloid Interface Sci. 2018, 512, 272-281.

19. Sharma, R.; Uma; Singh, S.; Verma, A.; Khanuja, M. Visible light induced bactericidal and photocatalytic activity of hydrothermally synthesized $\mathrm{BiVO}_{4}$ nano-octahedrals. J Photoch. Photobio. B. 2016, 162, 266-272.

20. Regmi, C.; Dhakal, D.; Lee, S.W. Visible-light-induced $\mathrm{Ag} / \mathrm{BiVO}_{4}$ semiconductor with enhanced photocatalytic and antibacterial performance. Nanotechnology. 2018, 29, 064001.

21. Napsucialy-Mendivil, S.; Alvarez-Venegas, R.; Shishkova, S.; Dubrovsky, J.G. 
ARABIDOPSIS HOMOLOG of TRITHORAXI (ATXI) is required for cell production, patterning, and morphogenesis in root development. J.Exp Bot. 2014, 65, 6373-6384.

22. Kong, X.; Tian, H.; Yu, Q.; Zhang, F.; Wang, R.; Gao, S.; Xu, W.; Liu, J.; Shani, E.; Fu, C.; Zhou, G.; Zhang, L.; Zhang, X. Ding, Z. PHB 3 maintains root stem cell niche identity through ROS-responsive AP2/ERF transcription factors in Arabidopsis. Cell Reports. 2018, 22, 1350-1363.

23. Tanaka, J.; Kiyoshi, K.; Kadokura, T.; Suzuki, K.I.; Nakayama, S. Elucidation of the enzyme involved in 2,3,5-triphenyl tetrazolium chloride (TTC) staining activity and the relationship between TTC staining activity and fermentation profiles in Saccharomyces cerevisiae. J. Biosci. Bioeng. 2020. https://doi.org/10.1016/j.jbiosc.2020.12.001.

24. Zhao, Y.; Xing, L.; Wang, X.; Hou, Y.; Gao, J.; Wang, P.; Duan, C.; Zhu, X.; Zhu, J. The ABA receptor PYL8 promotes lateral root growth by enhancing MYB77-dependent transcription of auxin-responsive genes. Sci Signal. 2014, 7 , ra53.

25. Wang, L.; Gong, L.; Zhao, E. Yu, Z.; Torimoto, Y.; Sadakata, M.; Li, Q.X. Inactivation of Escherichia coli by $\mathrm{O}^{-}$water. Lett. Appl. Microbiol. 2007, 45, 200-205.

26. Williams, J. S.; Kunkel, T. A. Ribonucleotides in DNA: Origins, repairand consequences. DNA Repair. 2014, 19, 27-37.

27. Chouchani, E. T.; Pell, V. R.; Gaude, E. Aksentijevic', D.; Sundier, S.Y.; Robb, E.L.; Logan, A.; Nadtochiy, S.M.; Ord, E. N. J.; Smith, A. C.; Eyassu, F.; Shirley, R.; Hu, C.H.; Dare, A. J.; James, A.M.; Rogatti, S.; Hartley, R.C.; Eaton, S.; Costa, A.S. H.; Brookes, P.S.; Davidson, S.M.; Duchen, M. R.; Saeb-Parsy, K.; Shattock, M.J.; Robinson, A.J.; Work, L.M.; Frezza, C.; Krieg, T.; Murphy, M. P.Ischaemic accumulation of succinate controls reperfusion injure through mitochondrial ROS. Nature. 2014, 515, 431-435.

28. Xie, L.; Chen, F.; Zou, X.; Shen, S.; Wang, X.; Yao, G.; Xu, B. Graphene oxide and $\mathrm{ABA}$ cotreatment regulates root growth of Brassica napus $\mathrm{L}$. by regulating IAA/ABA. J Plant Physiol. 2019, 240, 153007.

29. Begum, P.; Fugetsu, B. Phytotoxicity of multi-walled carbon nanotubes on red spinach (Amaranthus tricolor L.) and the role of ascorbic acid as an antioxidant. J Hazard Mater.2012, 243, 212-222.

30. Marslin, G., Sheeba, C. J., Franklin, G. Nanoparticles alter secondary metabolism in plants via ros burst. Front Plant Sci. 2017, 8, 1-8.

31. Melo, G.S.R.D.; Constantin, R.P.; Abraho, J.; Foletto-Felipe, M. D. P.; Constantin, R.P.; Santos, W. D.D.; Ferrarese-Filho,O.; Marchiosi, R. Titanium dioxide nanoparticles induce root growth inhibition in soybean due to physical damages. Water Air Soil Poll, 2021, 232, 25.

32. Xiang, Z. B.; Wang, Y.; Yang, Z.Q.; Zhang, D. Heterojunctions of $\beta-\mathrm{AgVO}_{3}$ $/ \mathrm{BiVO}_{4}$ composites for enhanced visible-light-driven photocatalytic antibacterial activity. J. Alloys Compd., 2019, 776, 266-275.

33. Hu, W.; Peng, C.; Luo, W.; Lv, M.; Li, X.; Li, D.; Huang, Q.; Fan, C. 
Graphene-based antibacterial paper. Acs Nano. 2010, 4, 4317-4323.

34. Liu, S.; Hu, M.; Zeng, T.H.; Wu, R.; Jiang, R.; Wei, J.; Wang, L.; Kong, J.; Chen, Y. Lateral dimension-dependent antibacterial activity of graphene oxide sheets. Langmuir. 2012, 28, 12364-12372.

35. Liu, S.; Zeng, T.H.; Hofmann, M.; Burcombe, E.; Wei, J.; Jiang, R.; Kong, J.; Chen, Y. Antibacterial activity of graphite, graphite oxide, graphene oxide, and reduced graphene oxide: membrane and oxidative stress. Acs Nano. 2011, 5, 6971-6980.

36. Maruri-López, I.; Jiménez-Bremont, J.F. Hetero- and homodimerization of Arabidopsis thaliana arginine decarboxylase AtADC1 and AtADC2. Biochem. Biophys. Res. Commun. 2017, 484, 508-513.

37. Gurung, S.; Cohen, M.F.; Fukuto, J.; Yamasaki, H. Polyamine-induced rapid root abscission in Azolla pinnata. J Amino Acids. 2012, 2012, 493209.

38. Peng, Y.; Chen, L.; Lu, Y.; Ma, W.; Tong, Y.; Li, Y. DAR2 acts as an important node connecting cytokinin, auxin, SHY2 and PLT1/2 in root meristem size control. Plant Signaling Behav. 2013, 8, e24226.

39. Eysholdt-Derzsó, E.; Sauter, M. Root bending is antagonistically affected by hypoxia and ERF-mediated transcription via auxin signaling. Plant Physiol. 2017, 175, 412-423.

40. Xu, H.; Feng, Y.; Fan, T.; Lü, T.; Xie, C.; Zhou, Y.; Tian, C. Disruption of IQM3 reducde the number of lateral roots and increases the length of primary root in Arabidopsis seedlings. Plant. Physiol J. 2019, 55, 629-634.

41. Okushima, Y.; Overvoorde, P.J.; Arima, K.; Alonso, J.M.; Chan, A.; Chang, C.; Ecker, J.R.; Hughes, B.; Lui, A.; Nguyen, D.; Onodera, C.; Quach, H.; Smith, A.; Yu, G.; Theologis, functional genomic analysis of the AUXIN RESPONSE FACTOR gene family members in Arabidopsis thaliana: Unique and overlapping functions of ARF7 and ARF19. Plant Cell. 2005, 17, 444-463. 